\title{
LARGE DIAMETER REDUCED FIELD TE01 TRAVELING WAVE WINDOW FOR X-BAND
}

\author{
W. R. Fowkes, R. S. Callin, E. N. Jongewaard, S. G. Tantawi \\ Stanford Linear Accelerator Center, Stanford University, Stanford, CA 94309 USA
}

\begin{abstract}
A single overmoded sized window is being considered for use on the SLAC 75 MW X-Band Klystron. The $65 \mathrm{~mm}$ diameter window operates in the circular TE01 mode in the reduced field, traveling wave configuration. Double step transitions were designed using MLEGO modematching program to suppress conversion to TE02. This compact design has a maximum peak rf electric field of $3.4 \mathrm{MV} /$ meter at $75 \mathrm{MW}$. Design details and high power test results are reported.
\end{abstract}

\section{INTRODUCTION}

The high power window presently used on all of the SLAC X-band klystrons has been reported on previously [1]. It is usually referred to as a $\mathrm{TE}_{01}$ reduced field traveling wave window. A pair of symmetrically located inductive irises are used to set up a standing wave in the regions between each iris and each $47 \mathrm{~mm}$ diameter ceramic window surface. This results in "forcing" a lower impedance at the window surface that exactly matches the characteristic impedance inside the ceramic thereby causing a pure traveling wave condition inside the ceramic. This design has been tested successfully to over $100 \mathrm{MW}$ in a traveling wave resonator (TWR) and has been serving as a single output window on the $50 \mathrm{MW}$ XL-4 series klystrons. This design however, is considered marginal at this power level.

The new $75 \mathrm{MW}$ periodic permanent magnet (PPM) focussed klystron presently uses a pair the windows described above, each passing $37.5 \mathrm{MW}$. Four $\mathrm{TE}_{10} / \mathrm{TE}_{01}$ mode transducers are presently required-an expensive luxury due to the cost of the mode transducers.

\section{SINGLE WINDOW REQUIREMENT}

A single window alternative has been sought that will safely handle the full $75 \mathrm{MW}$. The $47 \mathrm{~mm}$ TW window described above is considered to be very reliable up to a peak power level of about $40 \mathrm{MW}$ where the peak rf electric field at the window is $3.4 \mathrm{MV} /$ meter. Using this electric field level as a design criterion for a single 75 MW window, an increase in diameter to $65 \mathrm{~mm}$ would be required. This assumes of course that $\mathrm{TE}_{01}$ mode purity is maintained and the reduced field TW scheme is used. A problem that must be addressed in all all overmoded diameter circular windows is mode conversion.

"Work supported by Department of Energy Contract DE-AC0376SF00515
$\mathrm{TE}_{11}$ windows are susceptable to conversion to $\mathrm{TM}_{11}$, $\mathrm{TE}_{12}$ and $\mathrm{TM}_{12}$ in the transitions (tapered or stepped) going from single moded to overmoded diameters. $\mathrm{TE}_{01}$ windows are not as vulnerable to mode conversion with increases in diameter providing the mode converter has launched $\mathrm{TE}_{01}$ with good purity. Gradual tapers maintain $\mathrm{TE}_{01}$ mode purity but are not usually very compact. Stepped transitions to larger diameters are normally susceptible to conversion to higher order $\mathrm{TE}_{0 \mathrm{n}}$ modes unless special attention is given to the step design.

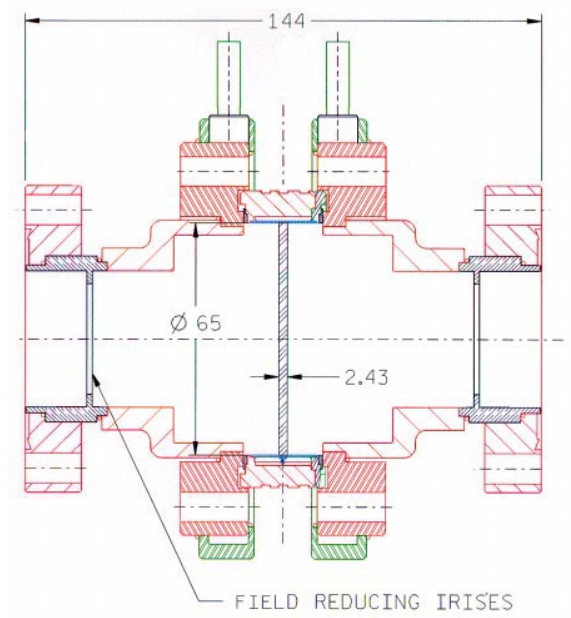

Figure 1. Compact overmoded $65 \mathrm{~mm}$ diameter TE01 window. Field reduction is accomplished by a combination of the 2-stage steps and irises. The double step is optimized to cancel $\mathrm{TE}_{02}$ that is created at each indivdual step.

\section{NEW TE01 TW WINDOW CONFIGURATION}

Here we describe a compact $65 \mathrm{~mm}$ diameter $\mathrm{TE}_{01}$ TW window that is virtually free of higher order modes. The maximum electric field at the surface of this window at $75 \mathrm{MW}$ is about the same as exists at for the previously described window at $40 \mathrm{MW} ; 3.4 \mathrm{MV} /$ meter. The $\mathrm{TE}_{01}$ mode is created in $38 \mathrm{~mm}$ diameter circular waveguide using the recently designed compact wrap-around mode transducer [2] shown in Fig 5. We are presently testing this window in the Traveling Wave Resonator and expect to reach a peak power level of $150 \mathrm{MW}$. At this writing power testing has been progressed towards higher levels 
The peak power achieved thus far is $100 \mathrm{MW}$.

The input and output ports for this window design are $38 \mathrm{~mm}$ in diameter. The transitions from $38 \mathrm{~mm}$ to $65 \mathrm{~mm}$ are two-stage steps designed using MLEGO@ which sets

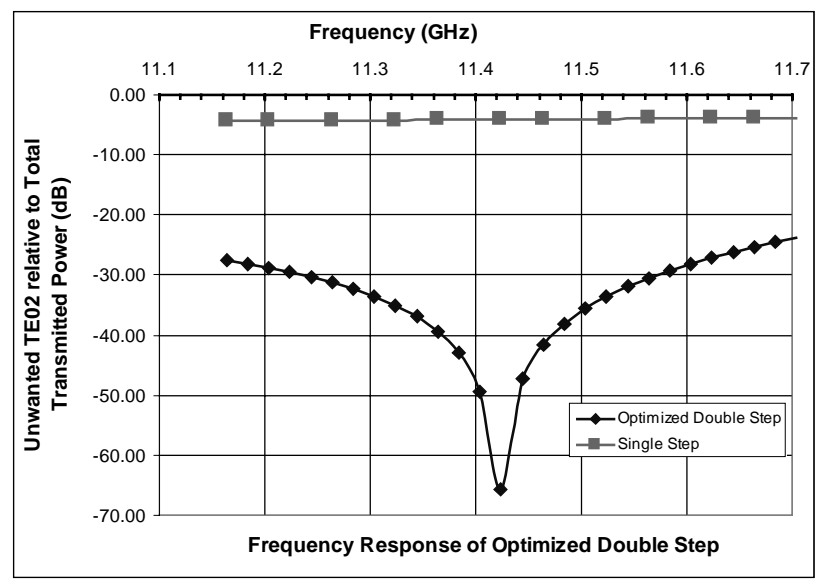

Figure 2. The above graph compares the conversion as a function of frequency of $\mathrm{TE}_{01}$ to unwanted $\mathrm{TE}_{02}$ for two types of stepped transitions; an optimized double-step and an abrupt single-step. The input and output diameters are $38 \mathrm{~mm}$ and $65 \mathrm{~mm}$ repectively.

up the cancellation of the $\mathrm{TE}_{02}$ mode excited at each step stage [3]. If one were to use a single sudden step in diameter from $38 \mathrm{~mm}$ to $65 \mathrm{~mm}$, the resulting mode conversion is the following: $\mathrm{S}_{21}$ of 0.78 for $\mathrm{TE}_{01}$ and 0.62 for $\mathrm{TE}_{02}$ with only pure $\mathrm{TE}_{01}$ exciting the smaller diameter port. By going to a two-step design, the length and diameter of the intermediate step is optimized to null out the natural conversion to $\mathrm{TE}_{02}$. The optimum intermediate diameter is $58.14 \mathrm{~mm}$ with a length of $19.48 \mathrm{~mm}$. The residual $S_{21}$ for $\mathrm{TE}_{02}$ drops to 0.00051 . The effectivness of this optimized step is shown in the graph in Fig. 2. It is compared with a single abrupt step as a function of frequency. The unwanted conversion from $\mathrm{TE}_{01}$ to $\mathrm{TE}_{02}$ is mimized for the operating frequency of $11.424 \mathrm{GHz}$.

All this is accomplished at the expense of a residual $\mathrm{TE}_{01}$ mismatch $\mathrm{S}_{11}$ of 0.167 in the $38 \mathrm{~mm}$ port. This residual mismatch, however, can be used to advantage in combination with an additional iris to set up the standing wave that is necessary to force the electric field down at the surface of the ceramic. The result is a pure traveling wave inside the ceramic.

It can be shown that the optimum VSWR needed to produce the TW condition within the ceramic is

$$
\sigma_{\text {opt }}=\frac{Z_{0}}{Z_{0}}=\frac{\lambda_{g}}{\lambda_{g}},
$$

and the total normalized iris susceptance that would give $\sigma_{\text {opt }}$ is given by

$$
\left|\frac{B}{Y_{0}}\right|=\frac{\lambda_{g}-\lambda_{g}{ }^{\prime}}{\sqrt{\lambda_{g} \lambda_{g}}}=1.3476
$$

where $\lambda_{\mathrm{g}}$ and $\lambda_{\mathrm{g}}$ ' are the guide wavelengths in the large diameter outside and inside the ceramic window respectively[1]. The double-step reflection of 0.167 corresponds to a normalized susceptance of 0.3388 . The difference is made up with symmetrical inductive irises each having a normalized susceptance of

$$
\left|\frac{B}{Y_{0}}\right|=1.3476-0.3388=1.0088
$$

located properly so that the stored energy between the iris and the 2-stage step is minimum. It is important that the irises be located in the smaller $(38.1 \mathrm{~mm})$ port since an inductive iris will convert to TE02 in the larger $(65 \mathrm{~mm})$ port.
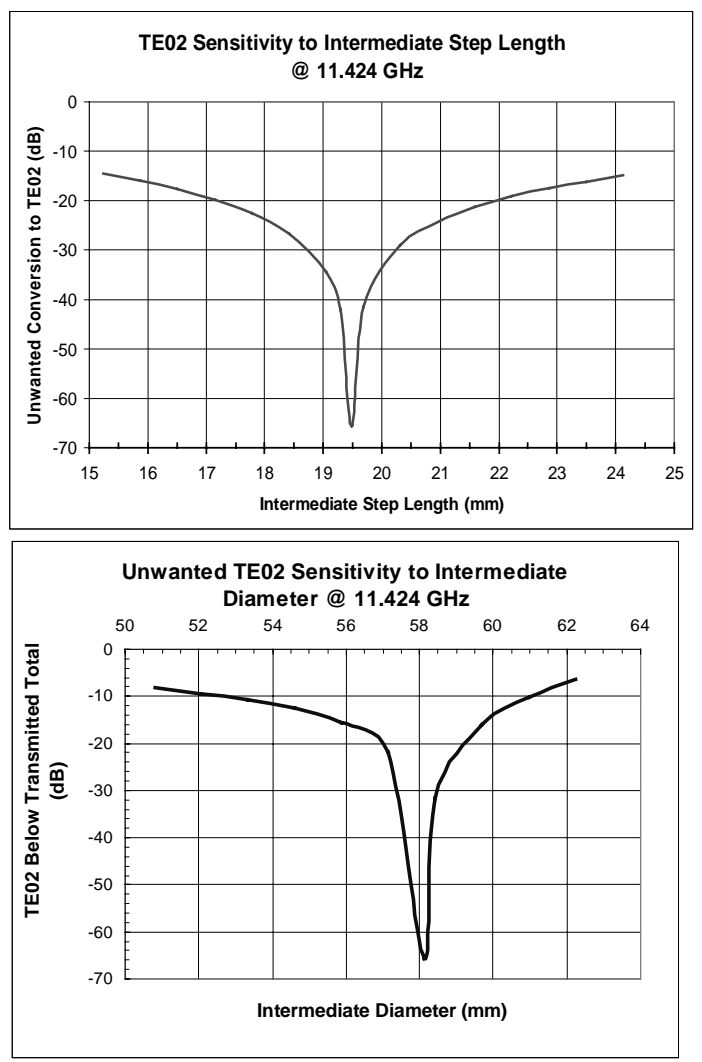

Figures $4 \mathrm{a}$ and $4 \mathrm{~b}$. The Double step from $38.1 \mathrm{~mm}$ to $65 \mathrm{~mm}$ has been optimized to nearly cancel any conversion to $\mathrm{TE}_{02}$ occurring at each step at the operating frequency of $11.424 \mathrm{GHz}$. This was accomplished using the mode matching code MLEGO. The variable parameters are the length and diameter of the intermediate step. The above graphs show the theoretical sensitivity of the conversion to unwanted $\mathrm{TE}_{02}$ to each of these parameters while the other is kept constant at the optimum value. 


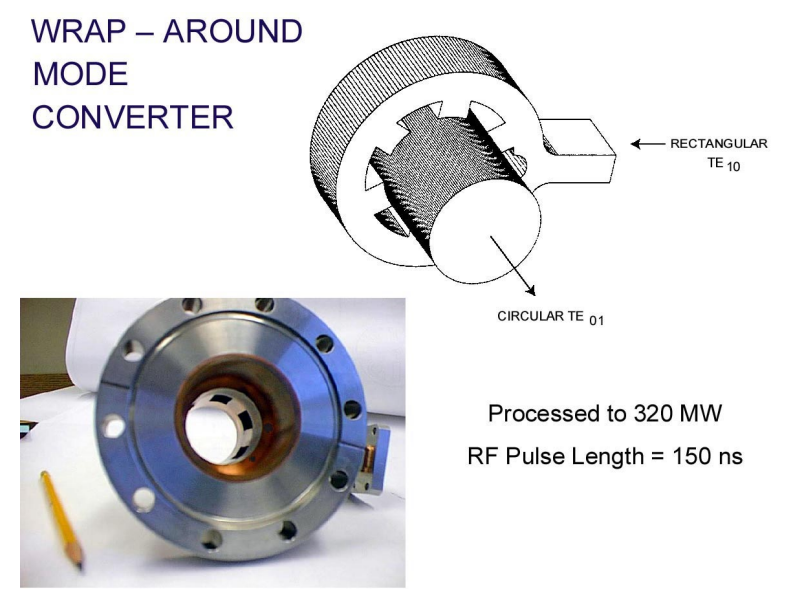

Figure 4. The new compact Wrap-around $\mathrm{TE}_{10} / \mathrm{TE}_{01}$ mode transducer that is used to launch a high purity $\mathrm{TE}_{01}$ mode in $38 \mathrm{~mm}$ circular waveguide. It has lower electric field and higher mode purity than the original "Flower Petal" mode transducer design.

\section{REFERENCES}

[1] W. R. Fowkes, R. S. Callin, S. G. Tantawi, and E. L. Wright, "Reduced Field TE01 X-Band Traveling Wave Window", Proceedings of 1995 Particle Accelerator Conference, Dallas, May 1995 pp 1587-9.

[2] S. G. Tantawi, "The Wrap-around Converter: A compact $\mathrm{TE}_{10} / \mathrm{TE}_{01}$ Mode Transducer" (to be published).

[3] MLEGO@ A mode matching code, Copyright S. G. Tantawi. 